\title{
VALORACIÓN BIOGEOGRÁFICA DEL BOSQUE MEDITERRÁNEO ESCLERÓFILO CON PALMERAS (Jubaea chilensis Mol (Baillon)) EN LA CUENCA DEL QUITEÑO (CHILE), A PARTIR DE LA APLICACIÓN DEL MÉTODO DE VALORACIÓN LANBIOEVA
}

\author{
Biogeographic evaluation of mediterranean sclerophyllous \\ forest with palm trees (Jubaea chilensis) in the Quiteño \\ river bassin, from the application of the LANBIOEVA \\ evaluation method
}

\author{
Victor G. Quintanilla Pérez ${ }^{1}$ y Pedro J. Lozano Valencia ${ }^{2, *}$ \\ ${ }^{1}$ Departamento Ingeniería Geográfica \\ Universidad de Santiago de Chile (USACH). victor.quintanilla@usach.cl \\ ${ }^{2}$ Departamento de Geografía, Prehistoria y Arqueología de la UPV/EHU. \\ Calle Tomás y Valiente S/N-Vitoria-Gasteiz (01006) \\ pedrojose.lozano@ehu.es \\ *Autor corresponsal
}

\begin{abstract}
Recibido: 30-07-2015. Aceptado: 15-10-2015. Fecha de publicación on-line: 06-07-2016
Citation / Cómo citar este artículo: Victor G. Quintanilla Pérez y Pedro J. Lozano Valencia (2016). Valoración biogeográfica del bosque mediterráneo esclerófilo con palmeras (Jubaea chilensis Mol (Baillon)) en la cuenca del Quiteño (Chile), a partir de la aplicación del método de valoración LANBIOEVA. Pirineos, 171, e018. doi: http://dx.doi.org/10.3989/Pirineos.2016.171002

RESUMEN: El artículo se basa en un trabajo de investigación desarrollado desde hace más de 20 años con el fin de consolidar un método de valoración biogeográfica de diferentes paisajes vegetales a escala global. Hace unos años, como consecuencia de una estancia de investigación, se valoraron diferentes unidades del ámbito mediterráneo de Chile. En los resultados llamaba la atención, sobremanera, el bosque mediterráneo esclerófilo con palmeras (Jubaea chilensis), ya que alcanzaba las puntuaciones más elevadas de este ámbito, y obtenía -además- el record absoluto hasta la fecha. El artículo se centra, expone y analiza los resultados obtenidos en la mencionada unidad, pero esta vez con inventarios y valoraciones sistemáticas realizadas en el año 2015. El área base de estudio se concentra en una pequeña microcuena: El Quiteño, del litoral montañoso de Viña del Mar. Los valores naturales y culturales no difieren de algunas formaciones del entorno, incluso de formaciones situadas en el ámbito europeo. Sin embargo, la prioridad de conservación se dispara teniendo en cuenta que las puntuaciones referidas al factor global de amenaza son muy elevadas.
\end{abstract}

PALABRAS CLAVE: Valoración biogeográfica; Quebrada el Quiteño; bosque mediterráneo esclerófilo; Jubaea chilensis; LANBIOEVA. 


\begin{abstract}
The present paper is based on research work that has been carried out for more than 20 years with the purpose of consolidating a method of biogeographic valuation of different plant scenes at a global scale. A few years ago, as a result of a research stay, different units of the Mediterranean environment of Chile were assessed. Among the results, the sclerophyllous Mediterranean forest with palms (Jubaea chilensis) clearly called one's attention because it not only achieved the highest scores in this setting, but it got the absolute record to date. The paper is centered on, presents and analyzes the results obtained in that unit, but this time with systematic inventories and assessments made in 2015. The base study area is concentrated in a small microbasin, El Quiteño, in the coastal mountains of Viña del Mar. The natural and cultural values do not differ from some formations of the surrounding, even from formations situated in the European setting, yet the conservation priority shoots up taking into account that the participations referred to the global threat factor are very high.
\end{abstract}

KEYWORDS: Biogeographic assessment; Quebrada el Quiteño; sclerophyllous Mediterranean forest; Jubaea chilensis; LANBIOEVA.

\section{Introducción}

En 2008 dos equipos, uno chileno y otro español, dieron lugar a la obtención de inventarios de distintas unidades de vegetación características de la región mediterránea chilena. Se trataba de poner en marcha, por el equipo español, el método de inventariado definido y, fundamentalmente, de valorar diferentes paisajes vegetales para comprobar la validez y robustez de la aplicación del método LANBIOEVA (Landscape Biogeographical Evaluation) fuera de los ámbitos en los que ya se había aplicado (Península Ibérica, Nicaragua, Península Escandinava, Balcanes...), con el fin de poder aplicarlo a la planificación y gestión de estos paisajes. Por falta del tiempo necesario, en aquella ocasión no se pudieron realizar inventarios sistemáticos y se optó por un inventariado selectivo centrado en parcelas muy concretas y que suponían una buena representación de la vegetación que se pretendía evaluar. La descripción y caracterización de estas formaciones ya fue abordada en un anterior trabajo (Quintanilla et al, 2012), de manera que no es el objetivo del presente artículo.

En la fase de valoración uno de aquellos paisajes obtuvo una puntuación muy superior, no sólo a la registrada por el resto de los paisajes vegetales de la región mediterránea chilena, sino de todos los ámbitos donde se había inventariado y valorado con anterioridad. Dicho trabajo dio lugar a una publicación (Lozano et al., 2015). Debido a dichos resultados, en 2014 se solicitó un proyecto de investigación titulado: "Caracterización y análisis, dinámica y valoración biogeográfica de la palma chilena Jubaea chilensis (Moll) Baillon en el bosque esclerófilo y fragmentado de Chile". Este proyecto es financiado por la DICYT de la Universidad de Santiago de Chile y el MECESUP del Ministerio de Educación. Dentro del primer gran objetivo a cumplir, se determinó la necesidad de un inventariado sistemático, estratificado y aleatorio que nos diera garantías de una valoración biogeográfica completa y objetiva. Dentro de este artículo se exponen los resultados no del inventariado, sino de la valoración de cada una de las parcelas estudiadas, lo que nos dará la valoración de un sininventario modelo o representativo del bosque mediterráneo esclerófilo con palmeras
(Jubaea chilensis) de la región mediterránea. Otro de los grandes objetivos persigue llamar la atención sobre el elevado valor biogeográfico de esta unidad que, en el sector analizado, no cuenta con ningún tipo de protección y se presenta, en cambio, muy amenazado por incendios forestales intencionados y sistemáticos y por la urbanización de amplios sectores donde el bosque mediterráneo esclerófilo de Jubaea chilensis (en adelante BMEJC) era la vegetación potencial hasta hace muy pocos años. Los ritmos de pérdida son tan elevados que, seguramente, en pocos años haya desaparecido de este sector. De hecho, toda la mancha de vegetación analizada se halla bajo diferentes grados de alteración, de manera que, en el mejor de los casos, estamos hablando de formaciones secundarias alteradas por diferentes razones.

Uno de los objetivos básicos de la Geografía y, como disciplina de la misma de la Biogeografía, es generar los conocimientos, herramientas metodológicas y resultados necesarios que aporten a la sociedad, no sólo conocimiento, sino también aplicación a los territorios y políticas de ordenación y gestión de los mismos. En los últimos siglos se asiste a una reducción drástica de determinados ecosistemas y, junto a ello, uno de los mayores problemas ambientales: la reducción de la diversidad a escala planetaria y la extinción de especies a un ritmo mucho más elevado que el estimado en tiempos pretéritos. Frente a los evidentes peligros mencionados, hace falta generar protocolos de análisis, valoración y diagnóstico ambiental, lo más objetivos posibles y que aporten un necesario cambio de paradigma hacia políticas territoriales, económicas, sociales y ambientales mucho más equilibradas y basadas en la verdadera capacidad de los ecosistemas de generar recursos. Se trata, por tanto, de planificar y gestionar los ecosistemas de forma responsable (McNeill, 2000).

De hecho, los territorios a conservar no sólo presentan valores medioambientales, sino también sociales, económicos, políticos, productivos, etc. para los que hay que procurar herramientas de valoración y gestión con visiones que vayan más allá de los simples valores naturales a conservar. Conscientes de esas necesidades, desde hace más de 20 años, se ha trabajado, a partir de distintos proyectos de investigación, en la línea de generar 
una herramienta potente y científicamente robusta que permita inventariar, analizar, diagnosticar, valorar y realizar las propuestas necesarias para la correcta gestión de diferentes paisajes, unidades ambientales o ecosistemas a escala global. Dicha propuesta se resume bajo el acrónimo LANBIOEVA (Landscape Biogeographical Evaluation).

Es interesante reseñar que la presente propuesta metodológica, de raigambre geográfica, constituye un modelo de aproximación al ejercicio valorativo concurrente y complementario con el de otras líneas de trabajo de enfoques muy diversos. Es el caso de trabajos como el de Costanza et al. (1997), que realizan una aproximación a la valoración de los servicios ambientales que ofrecen distintos ecosistemas desde la perspectiva de diferentes profesionales, no exenta de discusión puesto que valorar monetariamente algunos servicios naturales enfrenta poderosamente a la comunidad científica y técnica. Lo es, asimismo, el de la valoración de los ecosistemas y paisajes a través de los estudios cuantitativos relacionados exclusivamente con la biodiversidad (Whittaker, 1972), medida bajo tres parámetros - cantidad de especies, población de cada una de ellas y diversidad génica (Benton, 2001) - y que obvia criterios de índole cultural, frecuentemente tan importantes como los de orden natural en ecosistemas y paisajes profundamente manejados y modificados por la acción humana.

Frecuentemente, estos estudios se han centrado en ejercicios científicos relativamente complejos y difíciles de interpretar y utilizar por el gestor que, a la vista de las características físicas, ambientales, mesológicas, culturales, patrimoniales, percepcionales, etc., debe poner en marcha los preceptivos planes para la ordenación y gestión de los paisajes y territorios, fundamentalmente los de dominante natural. De ahí, que sean altamente interesantes las aproximaciones metodológicas que ofrezcan al mencionado gestor una herramienta operativa para la toma responsable de decisiones (Strijker et al., 2000; Debinski et al., 2001) y, sobre todo, las que ofrezcan una visión transversal que combine cuestiones relacionadas con los valores naturales intrínsecos de las unidades de paisaje y otros ligados a los procesos ecológicos, aspectos culturales y de manejo del territorio. Por todo ello, el método LANBIOEVA trata de ofrecer una metodología coherente, rigurosa, versátil y práctica, basada en pautas sencillas, flexibles y claras, con resultados estándar fáciles de aplicar e interpretar de cara a una correcta y jerárquica gestión de las comunidades y paisajes bióticos concernidos. Desde esta perspectiva, es un importante instrumento en la ordenación y gestión territorial, una herramienta fundamental para el conocimiento y la toma de decisiones respecto a los paisajes vegetales considerados como patrimonio natural y cultural. Como tal se está utilizando en la gestión tanto de las formaciones como de las especies que contienen.

El presente artículo recoge los resultados de inventariar y valorar, durante más de 7 meses durante el año 2015 y de manera sistemática, una serie de paisajes forestales de BMEJC, ubicados dentro de la Quebrada El Qui- teño. En definitiva, el objetivo es presentar los resultados de la aplicación del mencionado método (LANBIOEVA) a la evaluación de los paisajes del bosque mediterráneo esclerófilo con palmeras (Jubaea chilensis) de la Quebrada de El Quiteño en Viña del Mar (Chile).

\section{Material y métodos}

\subsection{El área de estudio}

La microcuenca o Quebrada El Quiteño, junto con la quebrada vecina de las Siete Hermanas, forman parte del Santuario de la Naturaleza Palmar El Salto, ubicado en los $33^{\circ} 03^{\prime}$ S. y $71^{\circ} 32^{\prime} \mathrm{W}$, en la Comuna de Viña del Mar, Chile Central (Figura 1). La microcuenca de El Quiteño se localiza en la vertiente occidental de la Cordillera de la Costa, en Chile mediterráneo y en planicies litorales de la Región de Valparaíso. La microcuenca El Quiteño abarca una superficie de 166 ha y la de Siete Hermanas 54 ha (Möder et al., 1997). Estas dos quebradas presentan las mayores concentraciones de palmas chilenas. Se orientan de sur a norte, con altitudes que oscilan entre los 90 y 250 m.s.n.m., son angostas y con pendientes fuertes. Ambas presentan una red de drenaje que confluye en un solo curso de agua permanente que desemboca en el Estero Marga-Marga, que a su vez muestra una amplia variación de caudal estacional debido al régimen pluviométrico propio del clima mediterráneo, con precipitaciones concentradas en otoño e invierno (Flores \& Aguirre, 2008).

Hemos concentrado nuestro estudio en el palmar del Quiteño debido a varias razones: en primer lugar, allí mismo se llevó a cabo la investigación de 2008; en segundo lugar, por su indudable calidad y, por último, por el riesgo de incendios que registra. El último, de hecho, acaeció en febrero de este mismo año (Figura 2). Por otra parte, su ubicación y las amenazas a las que se encuentra expuesto requieren una llamada de atención urgente de cara a su correcta planificación y gestión, fundamentalmente para poner en marcha políticas de protección.

J. chilensis (Moll) Baillon es una palma endémica de Chile que ha sido descrita desde mediados del siglo XVI. Su distribución se encentra entre los $30^{\circ}$ y $35^{\circ}$ Lat. Sur, constituyendo bosques dispersos, debido a la secular explotación que el ser humano ha venido haciendo de sus semillas comestibles (coquitos) y de su savia para la obtención de la miel de palma. Por su valor ornamental se planta bastante en Chile aunque, en gran medida, fuera de su área regular de distribución.

El palmar estudiado estaba primitivamente situado en las colinas que circundan las ciudades de Valparaíso y Viña del Mar, en Chile central. De acuerdo con Grau (2004) señalamos que ha sufrido numerosos cambios negativos debido a la gigantesca y progresiva extensión de estas ciudades, hoy día conurbadas, que han ido colonizando las laderas de forma caótica e incontrolada. Esta presión humana sobre los palmares de Jubaea chilensis ha sido funesta para su desarrollo y conservación. 


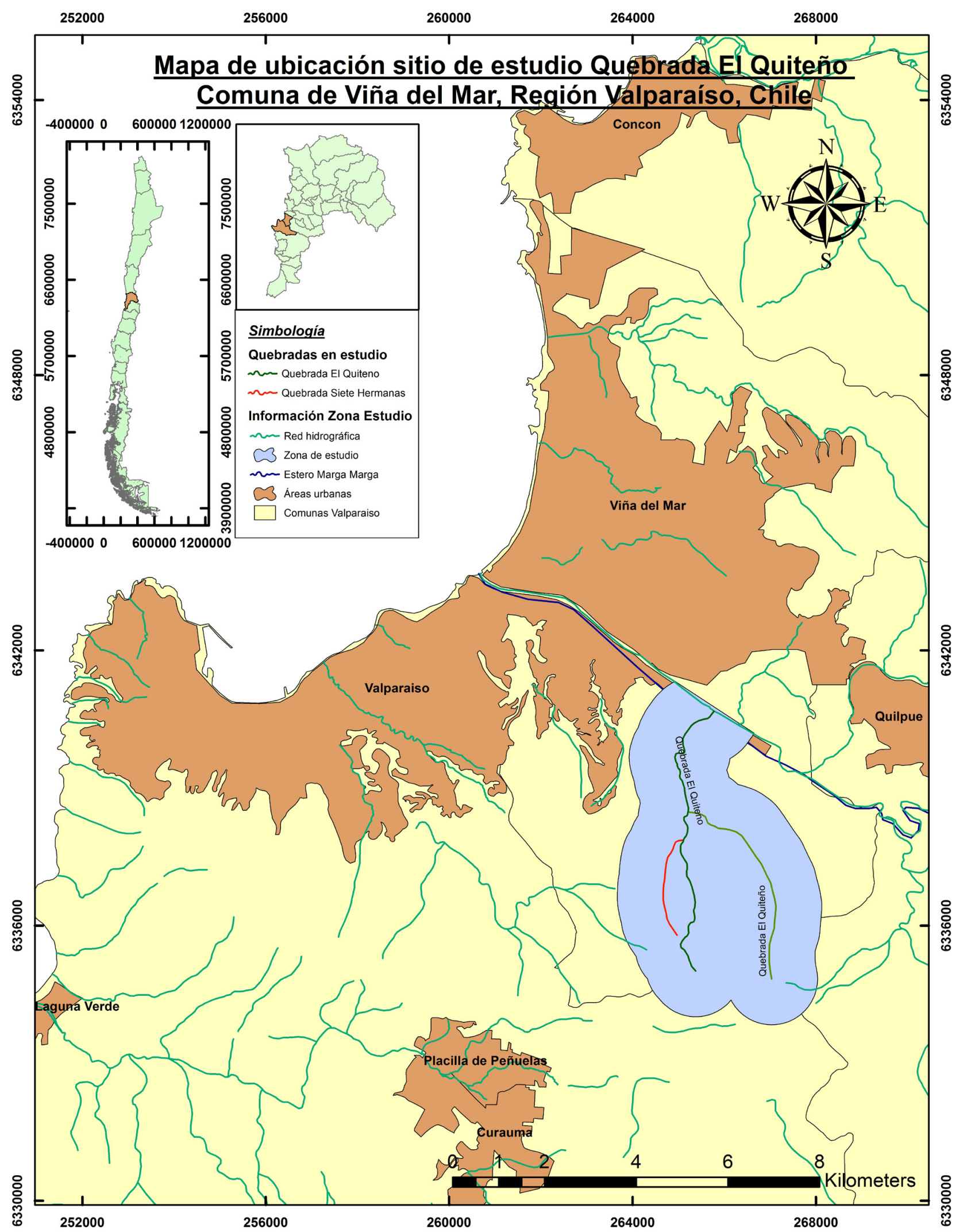

Figura 1: Área de estudio.

Figure 1: Study area. 
La estructura geológica del sector costero de Viña del Mar, donde se encuentra la Quebrada de El Quiteño, se caracteriza por presentar un basamento cristalino que se muestra como un bloque fracturado octogonalmente y, de hecho, las colinas de la Cordillera de la Costa se configuran como la mayor manifestación de estos alineamientos, en gran parte cubiertos de sedimentos del Plioceno de origen marino: formación Horcón (Álvarez, 1964). Respecto a otras características geológicas del sector, Álvarez (1964) señala que las terrazas ubicadas a ambos lados del Estero de Marga Marga son discordantes en altitud. Aquellas ubicadas al noreste son más bajas y alcanzan niveles entre los 200 y 240 m.s.n.m. Fueron labradas en rocas graníticas gneisicas de probable edad precámbrica y sobre parte de las cuales se depositaron los mencionados sedimentos marinos. El estero Marga Marga constituye una extensa falla geológica de dirección NW-SE.

En lo que concierne a la geomorfología (Figueroa et al., 1997) establecen que los cerros de la cuenca del Marga Marga están modelados en rocas graníticas de la llamada formación Quintay y del batolito de la costa, existiendo en el fondo del valle un relleno sedimentario derivado de la fracturación y erosión de las rocas de su entorno. En general éstas son granitoides metamórficas con variantes filonianas dispersas, de edades paleozoica y mesozoica, junto con depósitos sedimentarios del Pleistoceno.

Respecto a los suelos, según Luzio (2010) en estos microrelieves de costas destaca la formación de horizontes diagnósticos subsuperficiales y epidonesmólicos. Dominan las pendientes de 2 a $5 \%$ y 8 a $15 \%$, sin embargo en los sectores de lomajes y colinas son frecuentes las pendientes desde el $15 \%$ a más de $30 \%$. La profundidad de la parte superficial del suelo y el conjunto de horizontes (el solum) generalmente es superior a $1 \mathrm{~m}$ y sólo en algunos casos es ligeramente profundo (menos de $50 \mathrm{~cm}$ ). La clase textural del horizonte superficial varía desde franca a franco-arcillosa y en los horizontes más profundos va desde franco-arcilloso-limosa hasta

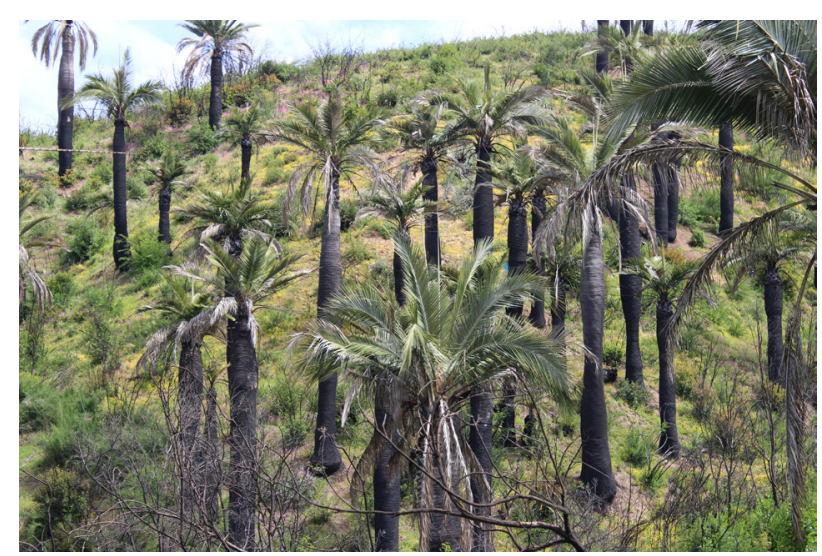

Figura 2: Foto de palmas quemadas por el incendio de febrero de 2015

Figure 2: Picture of palms burned by the fire of February 2015. franco-arcillosa. El pH del suelo sería ligeramente básico entre 7,19 a 7,35 (Grau, 2004). Debido a las fuertes pendientes, el suelo posee escasa materia orgánica y está muy expuesto a la erosión.

En lo que se refiere a las condiciones climáticas de estos relieves, predominan características de clima mediterráneo marino (Novoa \& Villaseca, 1989) de acuerdo con los datos meteorológicos más cercanos y que corresponden a la estación de Valparaíso (Figura 3). El régimen térmico se caracteriza por una temperatura media anual de $14,8^{\circ} \mathrm{C}$ con una máxima media del mes más cálido (enero) de $22,5^{\circ} \mathrm{C}$ y una mínima media del mes más frío (julio) de $8,3^{\circ} \mathrm{C}$. Según los autores antes citados el período libre de heladas es de 12 meses. La suma de temperaturas anuales, base $5^{\circ} \mathrm{C}$, es de 3,6 grados -días base$10^{\circ} \mathrm{C}$ de 1700 horas. Las horas de frío alcanzan a 220 acumuladas de Abril a Noviembre. La temperatura media anual se mantiene casi todo el año por encima de los $10^{\circ} \mathrm{C}$, lo cual es muy favorable.

Las lluvias suman $380 \mathrm{~mm}$. La evaporación anual es de $1350 \mathrm{~mm}$ y el mes de máxima evaporación es Enero, con $453 \mathrm{~mm}$ y un mínimo en Junio de $191 \mathrm{~mm}$. La estación seca empieza en Noviembre y termina en Abril, de manera que dura casi 7 meses.

En el pasado, estos palmares asociados al bosque esclerófilo, poseían poblaciones más extensas y densas pero la presión antrópica (crecimiento urbano, obras viales y explotación de las palmas) ha generado una disminución importante en su distribución. Otra causa muy importante en el retroceso de los palmares son los efectos de los incendios forestales que en estas colinas costeras de Viña del Mar y Valparaíso tienen una gran recurrencia histórica desde hace unos 35 años (Quintanilla \& Castro 1998; Quintanilla \& Reyes 1999; Quintanilla \& Castillo 2000; Quintanilla \& Morales 2013).

El bosque esclerófilo húmedo y mesotérmico predominante en los fondos de quebradas y en laderas de exposición sur, conforman la mayor parte del cortejo florístico acompañante de las comunidades de Jubaea chilensis (Moll) Baillon (1895). Los fuegos se inician sobre todo en laderas de suaves pendientes, que se encuentran preferentemente en exposición norte o en fondos planos donde son más constantes las comunidades de Quillaja saponaria, Lithraea caustica y Schinus latifolius acompañados de arbustos xéricos de laderas de solana donde predominan las comunidades de Flourensia thurifera, Colliguaja odorífera y Retanilla trinervia; junto a agrupaciones dominadas por Puya chilensis. Estas comunidades son altamente vulnerables a los incendios por su calidad de deciduas de verano y por el abundante estrato herbáceo. Fernández et al. (2009) y Quintanilla \& Castro (1998) comprobaron que luego de ocurrir el siniestro, gran parte de la vegetación quemada tiende a recuperarse, pero no se restablece la misma estructura y fisionomía del paisaje preexistente al fuego. Diversas causas antrópicas facilitan la generación de incendios desbastadores que, desde hace unos 15 años, se producen sucesivamente durante el verano en los cerros de Valparaíso y Viña del Mar. 


\subsection{Metodología utilizada}

La biogeografía aplicada trata, entre otras cosas, de evaluar cualitativamente la vegetación para estudiar su grado de conservación, suponiendo un instrumento fundamental para la ordenación y gestión territorial. El análisis valorativo de la vegetación constituye, por otra parte, el punto de encuentro de especialistas provenientes de distintos campos del conocimiento -botánicos, ecólogos, geógrafos, ingenieros y otros técnicos y profesionales (Cadiñanos \& Meaza, 1998a y 1998b)-, lo que hace deseable llegar a la elaboración de procedimientos consensuados y asumibles por la generalidad de los expertos.

\subsubsection{Inventariado}

Una vez predefinidas las unidades y su ubicación, a través de un laborioso trabajo de fotointerpretación y campo, se estimó un número mínimo de inventarios diseminados con carácter estratificado y aleatorio por el SIG (ARCGIS.10). A su vez, el número de inventarios a realizar para esta unidad, de cara a que de todos ellos se genere el sininventario tipo, depende de los siguientes criterios:

a) La superficie global que este tipo de paisaje ocupa dentro del área general. Cuanto mayor es ésta mayor cantidad de inventarios le corresponden.

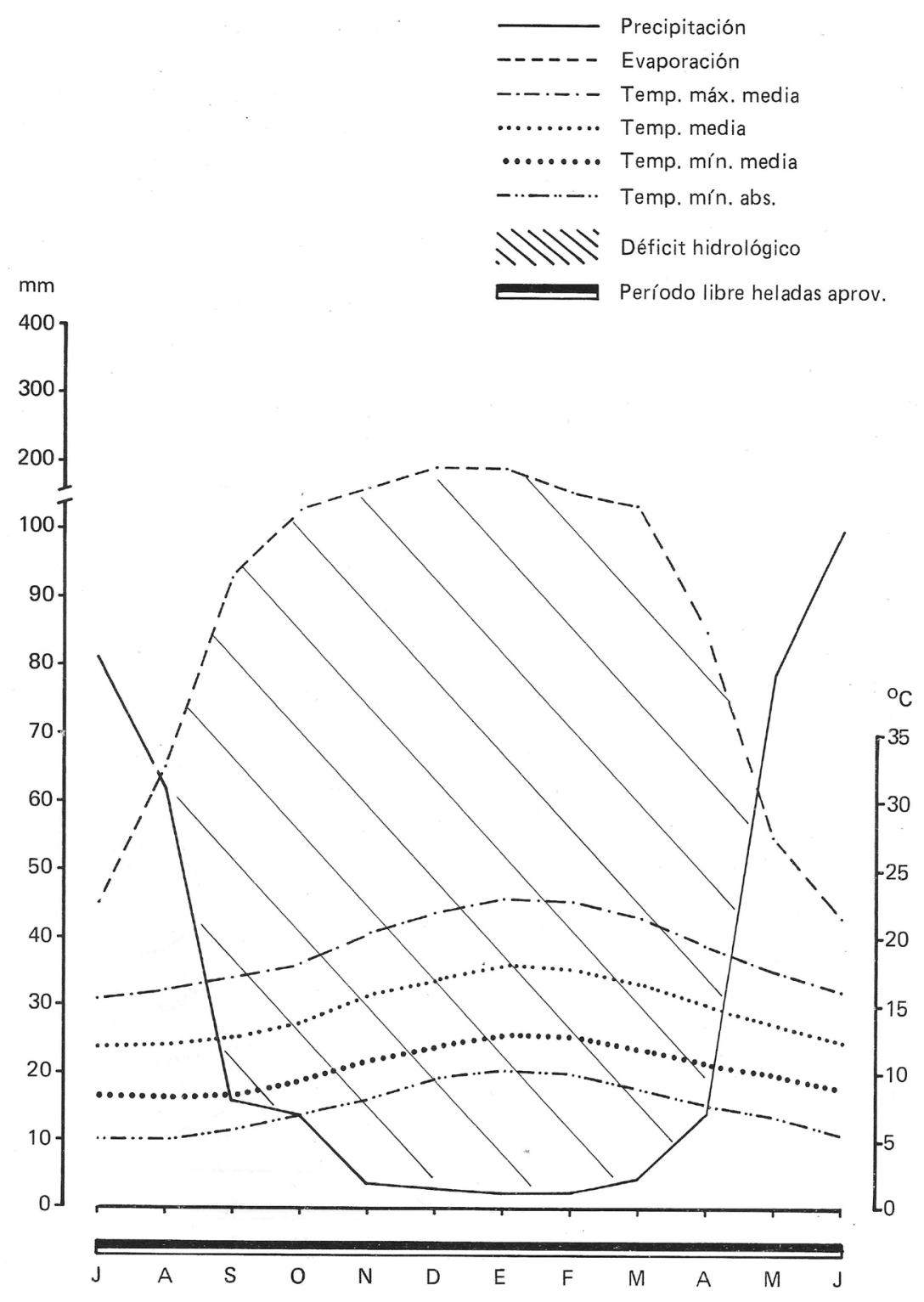

Figura 3: Clímodiagrama Valparaíso. Lat: $33^{\circ} 01^{\circ} \mathrm{S}$. Long: $71^{\circ} 039^{\circ} \mathrm{W}$. Alt: 41 m.s.n.m. Fuente: Novoa-Villaseca (1989).

Figure 3: Climodiagram Valparaíso. Lat: $33^{\circ} 01^{\circ}$ S. Long: $71^{\circ} 039^{\circ}$ W. Alt: 41 m.a.s.l. Fuente: Novoa-Villaseca (1989). 
b) Se tiene en cuenta, también, la cantidad de facies diferentes que puede presentar la unidad en cuestión. Cuanto mayor es el número de variantes mayor será el número de inventarios.

c) El número de inventarios se ha determinado, también, atendiendo al número de especies nuevas que aparecían entre los inventarios correlativos. De esta manera, si entre un inventario y los dos siguientes no existía un número superior al $5 \%$ de plantas nuevas, se estimaba que ese número de inventarios era suficiente.

Con todo, se han caracterizado y evaluado un total de 12 inventarios inéditos realizados entre diciembre de 2014 y junio de 2015. En primer lugar y, para cada uno de los inventarios realizados, se obtienen los datos de localización e identificación del lugar (coordenadas UTM, topónimos, etc.), aspectos y rasgos geográficos y medioambientales generales (topográficos, litológicos, geomorfológicos, edáficos e hidrológicos), fotografías de la parcela, etc. A continuación se tomaban no sólo los habituales datos sobre todos los taxones de la flora vascular presentes, sino también de la flora fúngica y liquénica (hongos y líquenes) y la cobertura y algunas especies de la briófita (estrato muscinal). Mientras estos últimos se valoraban por grupos y de forma general, las plantas vasculares se registraban con indicación de la cobertura de cada especie con una escala de 6 clases (5: máximo, +: mínimo), por cada uno de los cuatro estratos en que dividimos convencionalmente las comunidades (estrato $>5 \mathrm{~m}$, estrato entre 4,9 y $1 \mathrm{~m}$, estrato entre 0,9 y $0,5 \mathrm{~m}$ y estrato inferior $<0,5 \mathrm{~m}$ ) y la cobertura global.

Además se han tomado una serie de datos imprescindibles para la valoración complementaria de las comunidades forestales. Así, la cobertura global y la riqueza por estratos (COBEST y RIQUEST), la diversidad de hábitats no desglosables (FORHAB), la superficie de la mancha homogénea (FORESP), la variedad dasonómica tipológica (FORFIS), y los valores culturales y etnográficos añadidos (FORCUL).

Como es fácil advertir, estos inventarios requieren más tiempo que los fitosociológicos o similares. Ahora bien, una vez realizados, la información obtenida es mucho mayor, lo que permite su posterior utilización no sólo para la caracterización geobotánica del área de estudio en cuestión, sino también para reflejar su disposición estructural y biogeográfica, así como, en última instancia, para realizar la evaluación y gestión de la vegetación y el paisaje, objetivo fundamental del presente trabajo.

\subsubsection{Valoración biogeográfica}

En la figura 4 se pueden observar los diferentes criterios de valoración de forma gráfica.

El objetivo de este método es presentar, desde esa perspectiva integradora, una metodología valorativa coherente, rigurosa y práctica, basada en pautas sencillas, flexibles y claras. Supone, además, la última versión de un esfuerzo sostenido de años dirigido a perfeccionar procedimientos de valoración fiables y versátiles que tengan validez para el mayor número de ambientes posible y que puedan ser utilizados, indistintamente, por estudiosos de la ciencia de la vegetación, por expertos y profesionales y por las administraciones. La propuesta metodológica descansa en varios criterios valorativos diferenciados que constituyen, al tiempo, eslabones estrechamente ligados del sistema operativo. Por mor a la brevedad, no se explican las diferentes tipologías propias de cada criterio de valoración. Para ello nos remitimos a Cadiñanos \& Meaza (1998a).

A. El Interés Natural Global (INNAT): Compuesto por tres tipos de criterios que abarcan el interés fitocenótico, el interés territorial global y el interés mesológico global.

A.1. Interés Fitocenótico Global (INFIT): Los criterios fitocenóticos estiman caracteres intrínsecos de la vegetación y del paisaje tales como la diversidad, la naturalidad, la madurez y regenerabilidad espontánea. La madurez, al contar con una mayor importancia aparece con el doble de ponderación. Como consecuencia, la unidad valorada puede obtener un INFIT que puede variar entre 5 y 50 puntos siguiendo la siguiente fórmula:

$$
\begin{aligned}
& \text { INFIT }=\text { DIV }(1 \text { a } 10)+\text { NAT }(1 \text { a } 10)+ \\
& + \text { MAD }(2 \text { a } 20)+\operatorname{REG~}(1 \text { a } 10) .
\end{aligned}
$$

A.2. Interés Territorial Global (INTER): Los criterios territoriales son bifactoriales -se aplican tanto a nivel de especie como de agrupación- y consideran los atributos de rareza, endemismo, relictismo y carácter finícola, tanto de los taxones presentes como de la propia formación o unidad de paisaje. La rareza, al contar con una mayor importancia, aparece con el doble de ponderación. Consecuencia de ello, la unidad valorada puede obtener un INTER que puede variar entre 0 y 50 puntos siguiendo la siguiente fórmula:

$$
\begin{aligned}
& \text { INTER }=\text { RAR }(0 \text { a } 20)+\text { END }(0 \text { a } 10)+ \\
& + \text { REL }(0 \text { a } 10)+\text { FIN }(0 \text { a } 10) .
\end{aligned}
$$

A.3. Interés Mesológico Global (INMES): Los criterios mesológicos evalúan la contribución de la vegetación a la protección, equilibrio y estabilidad de la biocenosis, el hábitat y el geo-biotopo en el que radica. En su virtud, se proponen 5 parámetros, correspondientes a las funciones geomorfológica, climática, hidrológica, edáfica y faunística (Cadiñanos \& Meaza, 1998a). La función geomorfológica, al contar con una mayor importancia, aparece con el doble de ponderación. Consecuencia de ello, la unidad valorada obtiene un INMES que puede variar entre 6 y 60 puntos siguiendo la siguiente fórmula:

INMES $=$ GEO $(2$ a 20$)+$ CLIM $(1$ a 10$)+$

$+\operatorname{HIDR}(1$ a 10$)+\operatorname{EDAF}(1$ a 10$)+$ FAU $(1$ a 10$)$. 


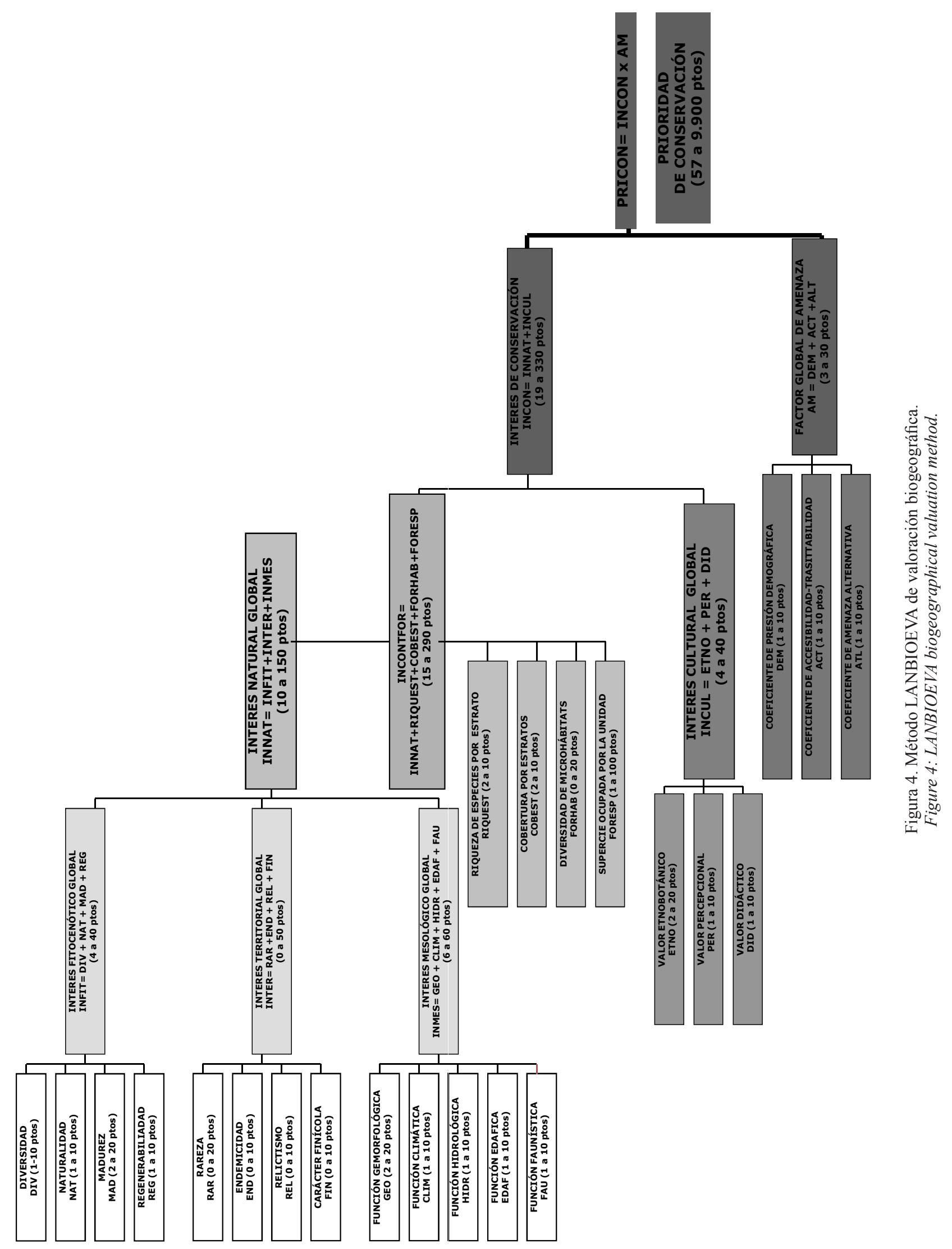

Pirineos, Vol. 171, Enero-Diciembre, 2016, e018. ISSN-L: 0373-2568, doi: http://dx.doi.org/10.3989/Pirineos.2016.171002 
La suma de estos tres criterios da lugar al denominado Interés Natural Global (INNAT) y, por tanto, puede ser utilizado como un criterio de raigambre y naturaleza puramente ambiental. En cualquier caso, dicho parámetro puede oscilar entre 10 y 150 puntos. Su fórmula sería:

$$
\begin{aligned}
\text { INNAT }= & \text { INFIT }(4 \text { a } 40)+\operatorname{INTER}(0 \text { a } 50)+ \\
& + \text { INMES }(6 \text { a } 60) .
\end{aligned}
$$

B. El Interés Natural Forestal (ITNATFOR): A los valores naturales se le suman parámetros relacionados con la cobertura de los diferentes estratos del bosque (COBEST), la diversidad específica dentro de cada estrato (RIQEST), la superficie continua de la unidad estudiada (FORESP) o la diversidad de microhábitats (FORHAB). El ITNATFOR puede oscilar entre 5 y 140 puntos.

C. Valoración del Interés Cultural (INCUL): Los criterios de carácter cultural han sido obviados o infrautilizados en la mayor parte de las propuestas valorativas, debido básicamente al reduccionismo naturalístico. Sin embargo, concitan una atención cada día mayor en la sensibilidad y políticas conservacionistas. El INCUL se calcula teniendo en cuenta, a su vez, otros tres valores diferentes.

C.1. Valor Etnobotánico (FORETNO): Este criterio trata de evaluar los aspectos etnoculturales (históricos, arqueológicos, religiosos, mitológicos, simbólicos, recreativos, medicinales...) de las plantas, la vegetación y el paisaje que, en su caso, pueden contribuir a hacerlas acreedoras de conservación: vestigios, estructuras y microtopografías relictuales de prácticas forestales (morfología de fustes y ramaje; muros, lezones, setos, caballones y cárcavas de contención o de separación de parcelas; carboneras...), agroganaderas o preindustriales (ferrerías, molinos, aceñas, batanes...) configuradoras de paisajes vegetales peculiares. Se recomienda adjudicar 1 punto por cada elemento considerado de alto valor etnobotánico, respetando siempre la escala de 1 a 10 puntos. El FORETNO es multiplicado por un factor de corrección de 2, puesto que cuenta con mayor importancia que los otros tres, de manera que puede fluctuar entre 0 y 20 (Cadiñanos \& Meaza, 1998a).

C.2. Valor Percepcional (PER): Es un parámetro que trata de valorar la relación perceptiva (escénica, estética, incluso vivencial) del hombre con respecto a la vegetación. Para su correcta evaluación, lo ideal es contar con encuestas objetivas de preferencias, gustos, querencias y afinidades. El PER fluctúa entre 1 y 10 puntos (Cadiñanos \& Meaza, 1998a).

C.3. Valor Didáctico (DID): Este criterio trata de aquilatar el interés pedagógico del paisaje en sus aspectos naturales y culturales y en la educación y concienciación ambiental de la población en general. Se propone la utilización de la siguiente escala genérica que el investigador habrá de aplicar usando pautas previamente establecidas: desde el valor 1 a aquellas unidades que cuenten con un valor didáctico muy bajo hasta el 10 a aquellas que obtengan uno de muy alta estima. De esta forma el DID puede fluctuar entre 1 y 10 (Cadiñanos \& Meaza, 1998a).
Con todo, el Interés Cultural (INCUL) deriva de la suma de las calificaciones adjudicadas a los 3 criterios valorativos que lo integran. Esto es:

$$
\begin{aligned}
& \text { INCUL = ETNO (2 a } 20)+ \\
& \text { PER (1 a 10) + DID (1 a 10) }
\end{aligned}
$$

El interés cultural global oscila, entonces, entre 4 y 40 puntos.

D. El interés de conservación de una determinada agrupación vegetal o paisaje (INCON) resulta de sumar a la puntuación de INNATFOR (10 a 150) la calificación obtenida por ITNATFOR (5 a 140) más el INCUL (14 a 190), con lo que el rango de INCON oscila entre 19 y 330 puntos.

E. La Prioridad de conservación (PRICON). Como se puede apreciar en los siguientes párrafos es solidaria pero, al tiempo, sustancialmente diferente a la de INCON (interés de conservación), ya que incluye consideraciones ajenas, extrínsecas, a este último. Su resultado ha de ser asumido de manera independiente y no debe ser confundido con él. La prioridad de conservación está, pues, expresamente ideada para su utilización por la administración competente o el gestor, quienes precisan de un diagnóstico claro y operativo sobre cuáles son los espacios que deben ser priorizados cara a su protección y cuáles pueden esperar.

El grado de amenaza que pesa sobre las unidades de vegetación o paisajes concernidos en el proceso evaluativo se calibra en función de tres parámetros: presión demográfica, accesibilidad-transitabilidad y amenaza alternativa.

E.1. El Coeficiente de Presión Demográfica (DEM): introduce la variable demográfica humana en el sistema valorativo. En su virtud, se priman o penalizan situaciones de alta o baja densidad de población, con mayor o menor peligro, respectivamente, de alteración de la vegetación. La escala a aplicar se obtiene en función de los rangos de densidad real en habitantes $/ \mathrm{km}^{2}$ de la zona de estudio. El investigador debe tener en cuenta cuestiones como la demografía de la zona, cercanía a grandes núcleos de población y conurbaciones y flujos estacionales, así como la disponibilidad y nivel de detalle de las fuentes estadísticas. La escala propuesta varía entre el 1 para aquellos ámbitos con densidades de menos de 50 habitantes por $\mathrm{km}^{2}$ hasta 10 en aquellos que se superen los 450 hab. $/ \mathrm{km}^{2}$.

E.2. El Coeficiente de Accesibilidad-Transitabilidad $(\mathrm{ACT})$ es un parámetro de atención inexcusable a la hora de establecer el nivel de amenaza al que se encuentra expuesta la unidad de paisaje, puesto que la presencia e impronta del ser humano está condicionada por la topografía del terreno, la densidad, tamaño, estado de conservación y grado de penetración de la red viaria y por la estructura más o menos abierta de la unidad valorada; en su caso, también por las limitaciones impuestas por los propietarios o administradores del terreno o por normativa legal 
dictada por la Administración. La escala propuesta es la que muestra una matriz de doble entrada (6 valores de accesibilidad y otros 6 de transitabilidad desde muy baja hasta absoluta para las dos). La combinación de las dos variables va generando puntuaciones que varían desde el 1 hasta 10 cuando la accesibilidad y transitabilidad son absolutas.

E.3. Coeficiente de Amenaza Alternativa (ALT): se incluyen y calibran bajo este concepto factores alternativos de amenaza que, eventualmente, puedan afectar a la unidad de vegetación o el paisaje objeto de evaluación de manera grave, real y coetánea al ejercicio valorativo $-\mathrm{O}$ a muy corto plazo-: catástrofes naturales o provocadas (inundaciones, fuegos), daños palpables por lluvia ácida, vertidos tóxicos o contaminantes, eutrofización, plagas u otras causas de mortalidad excesiva, invasión o desplazamiento de la vegetación original por plantas xenófitas agresivas, desaparición de la vegetación a corto plazo por talas masivas, acondicionamiento para infraestructuras, construcciones, tendidos eléctricos, depósitos, dragados, actividades extractivas, etc. La escala propuesta varía desde el 1 para la amenaza alternativa muy baja hasta el 10 para aquella que es muy alta.

Así, una vez obtenidos los tres coeficientes, se obtiene el Factor Global de Amenaza (AM) sumando los valores de los coeficientes demográfico (DEM=1-10), de accesibilidad-transitabilidad (ACT $=1-10)$ y de amenaza alternativa $(\mathrm{ALT}=1-10)$, con lo que el resultado de AM oscila entre 3 y 30 puntos.

La prioridad de conservación (PRICON) de una determinada agrupación vegetal o paisaje se determina multiplicando su valor de INCON (19 a 330) por el coeficiente $\mathrm{AM}$ (3 a 30) que le corresponda, con lo que el rango de PRICON oscila entre 57 y 9900 puntos.

\section{Resultados y discusión}

A partir de los inventarios realizados y, tal y como quedó patente en el capítulo anterior, se dio lugar a la valoración de cada uno de ellos. Para cada inventario, por tanto, se generó una valoración biogeográfica, de manera que las aquí expuestas son los resultados de cada uno de los parámetros valorados para los diferentes inventarios de cada parcela. No vamos a detenernos en la composición y estructura de las unidades estudiadas puesto que no es objeto de este estudio y además puede consultarse en Quintanilla et al. (2012). No obstante, a continuación, en la tabla 1 se puede comprobar los datos y características geográficas más importantes de cada una de las parcelas y sus respectivos inventarios.

Como se puede comprobar, existen cuatro facies básicas dentro de este paisaje forestal. En primer lugar estaría el bosque esclerófilo mediterráneo con las palmas, en segundo lugar la misma formación pero con la repoblación de eucaliptos, en tercer lugar el bosque esclerófilo mediterráneo con palmas pero, en este caso, con la repoblación a partir de eucaliptos y mimosas y, en último lugar, y aunque resulta extremadamente raro y difícil de hallar, se encontraría el mismo palmar con bosque esclerófilo mediterráneo pero sólo con repoblación de mimosa. En la tabla 2 aparecen recogidos cada uno de los parámetros valorados y su valor. En gris aparecen las diferentes sumas. En primer lugar las sumas parciales de los criterios fitocenológicos, territoriales y mesológicos, que dan lugar al valor natural total (INNAT). En un gris un poco más oscuro se añade la valoración complementaria de las masas forestales, dando lugar al INNATFOR global. A continuación se valoran los distintos parámetros o criterios culturales (con un gris todavía más oscuro) y se suman a los anteriores, de manera que obtenemos el INCONTFOR global (tiene el mismo tono gris que el anterior) y que no deja de ser una suma entre lo natural y lo cultural.

Tabla 1. Características geográficas de las parcelas estudiadas (COD.=Código; EXPO: Exposición; ALT.: Altitud en metros y PEND.: Pendiente en grados) (P.: Palmeral de Jubaea chilensis). Elaboración propia.

Table 1. Geographic characteristics of the studied plots (COD = code; EXPO = exposure; $A L T=$ altitude in metres; and PEND = slope in degrees) (P: palm grove of Jubaea chilensis). Own design.

\begin{tabular}{|l|l|l|l|c|c|}
\hline CÓD. & \multicolumn{1}{|c|}{ TIPO DE FORMACIÓN } & \multicolumn{1}{|c|}{ UTM } & \multicolumn{1}{|c|}{ EXPO } & ALT & PEND \\
\hline Q1 & P. con sotobosque esclerófilo & 6339939264202 & NW & 119 & 15 \\
\hline Q2 & P. con sotobosque esclerófilo & 6339724264257 & NW & 154 & 13 \\
\hline Q3 & P. con sotobosque esclerófilo & 6339478264284 & SE & 160 & 15 \\
\hline Q4 & P. con sotobosque esclerófilo & 6339589264292 & SE & 142 & 12 \\
\hline Q5 & P. con sotobosque esclerófilo & 6339864269270 & SE & 160 & 8 \\
\hline Q6 & P. con sotobosque esclerófilo & 6339702275077 & NE & 130 & 43 \\
\hline Q7 & P. con sotobosque esclerófilo y plantación de eucalipto & 6339699264924 & SE & 80 & 5 \\
\hline Q8 & P. con sotobosque esclerófilo y plantación de eucalipto & 6339631264936 & NW & 82 & 5 \\
\hline Q9 & P. con sotobosque esclerófilo y plantaciones de eucalipto y mimosa & 6339490264949 & SW & 84 & 5 \\
\hline Q10 & P. con sotobosque esclerófilo y plantaciones de eucalipto y mimosa & 6339984264972 & NW & 45 & 5 \\
\hline Q11 & P. con sotobosque esclerófilo y plantaciones de mimosa & 6339718264944 & SW & 70 & 4 \\
\hline Q12 & P. con sotobosque esclerófilo & 6339461264291 & SE & 112 & 32 \\
\hline
\end{tabular}


A continuación y, una vez obtenido el valor del interés de conservación (INCONTFOR), se valoran los criterios relacionados con el factor global de amenaza. Éste aparece con el gris más oscuro. El PRICON o prioridad de con- servación se obtendrá a partir de la multiplicación de los dos anteriores dando los valores finales (en negrita).

Los resultados muestran una clara jerarquía. En primer lugar y, tal y como podía preverse, los paisajes forestales

Tabla 2. Resultados de las valoraciones para cada uno de los criterios de cada inventario o parcela de estudio. La última columna recoge las medias. Elaboración propia.

Table 2. Results of the valuations of each of the criteria of every inventory or study plot. The last column shows the measurements. Own design.

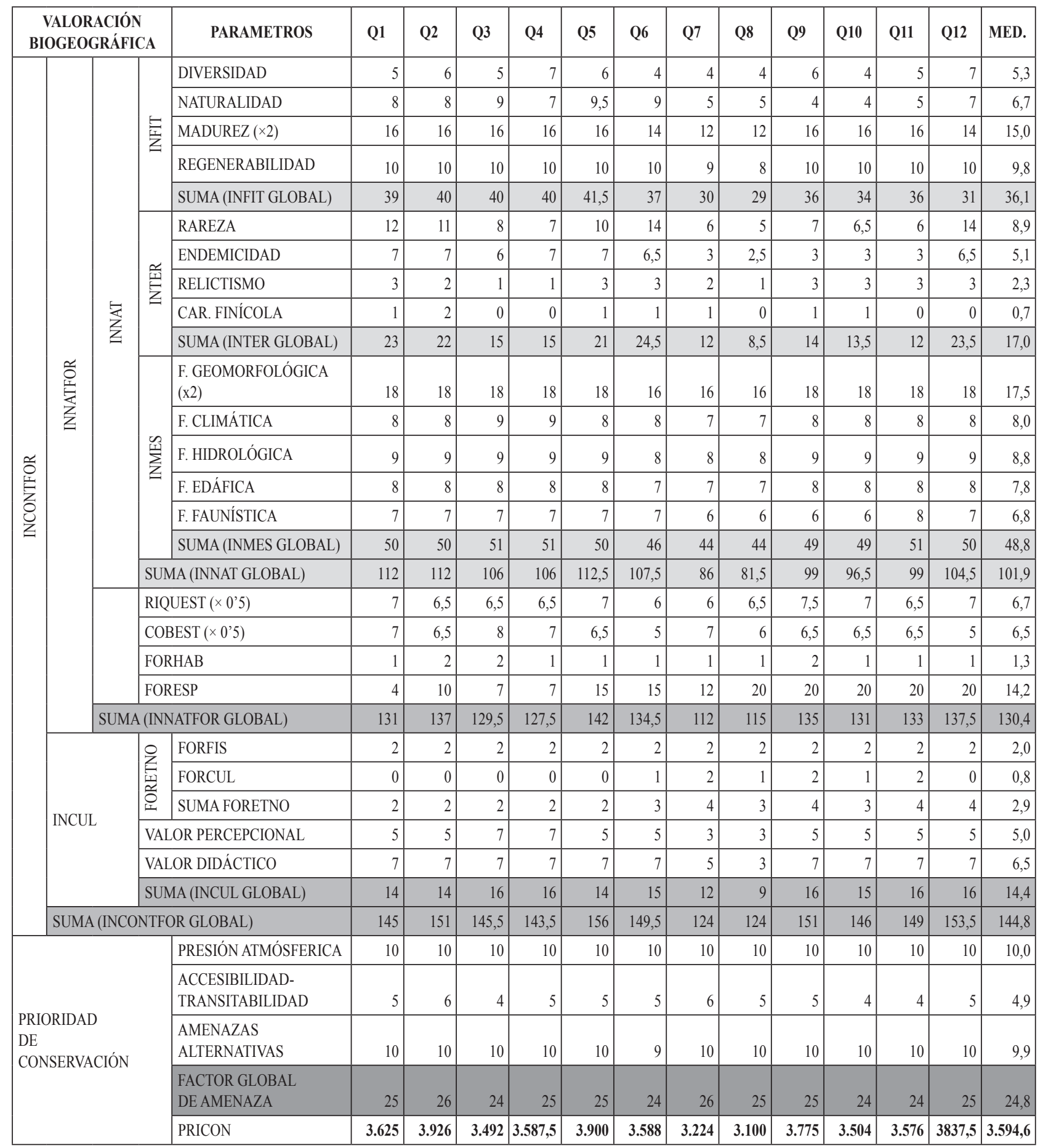


autóctonos o sin presencia de plantaciones con especies alóctonas o vestigios de las mismas, muestran valoraciones generales superiores a aquellos bosques con palmas pero con repoblaciones de eucalipto (Eucalyptus globulus) (Figura 5) o mimosa (Acacia dealbata) (Figura 6).

Haciendo un análisis más pormenorizado, se puede observar que dentro del primer grupo de criterios que dan lugar al interés fitocenótico global no existen excesivas diferencias. En cuanto a la diversidad específica, los inventarios se mueven entre los 4 y los 7 puntos, es decir, posiciones intermedias que traducen unas diversidades relativamente modestas entre las 28 especies registradas en Q12 y las 12 de Q7 y Q8. En realidad se trata de diferencias bastante abultadas teniendo en cuenta que son sólo facies del mismo paisaje. En lo que respecta a la naturalidad, este criterio oscila ostensiblemente derivado de la existencia de facies dominadas por la presencia de una o dos especies alóctonas con gran cobertura como son el eucalipto (Eucalyptus globulus) o la mimosa (Acacia dealbata). Por otra parte, la cantidad de neófitos es bastante alta teniendo en cuenta que, además, con coberturas más modestas existen neófitos como: Rubus ulmifolius, Teline monspessulana, Cytisis striatus, Nycotiana glauca o Fumaria officinalis. Esta gran abundancia de neófitos es todavía más preocupante si se tiene en cuenta que muchos de ellos se encuentran absolutamente naturalizados, colonizando importantes sectores y compitiendo con especies autóctonas.

Por su parte, en cuanto a la madurez, las distintas parcelas tienen una variación tan sólo de 3 puntos (que se convierten en 6 al ser este criterio multiplicado por 2). En realidad las parcelas oscilan entre los 6 puntos que les corresponden a los matorrales de porte alto con algún pie arbóreo (Q7 y Q8), el piroclimax boscoso o plagioclimax (Q6 y Q12) y, la máxima puntuación queda registrada en el resto de parcelas que se corresponde con un paraclimax. En ningún caso existe una parcela que cuente con los 20 puntos relacionados ya con etapas climácicas o

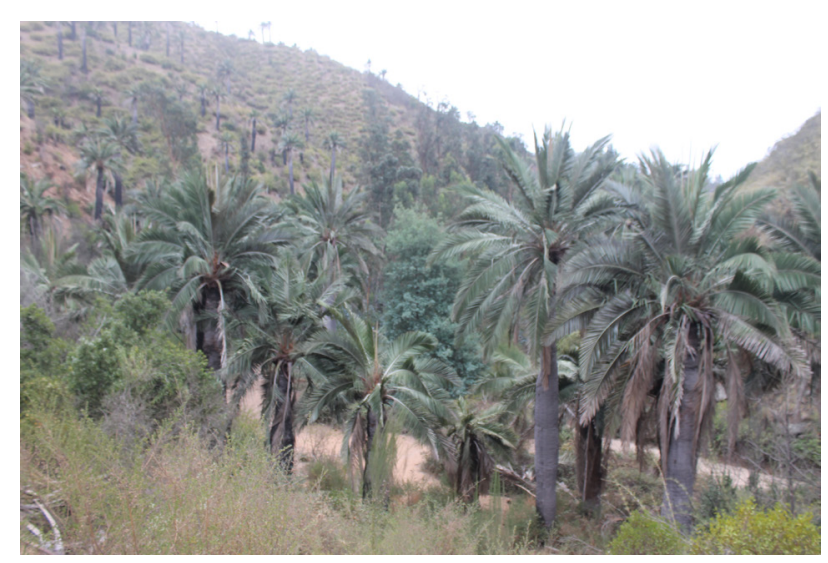

Figura 5: Foto de Jubaea chilensis acompañada con repoblaciones de Eucaliptus globulus.

Figure 5: Picture of Jubaea chilensis together with repopulations of Eucalyptus globulus. postclimácicas. Por último, para este grupo de criterios, la regenerabilidad o resiliencia ecológica, registra diferencias todavía más modestas. En general, la mayor parte de las parcelas responde a una unidad relictual con grandes dificultades en su recuperación y con turnos o ciclos sucesionales relativamente prolongados en el tiempo, 10 de las 12 parcelas cuentan con la máxima puntuación (10), mientras las parcelas Q8 y Q7 cuentan con 8 y 9 puntos respectivamente y responden a facies de vegetación más cercana al climax en medios xericos pero sin llegar a las facies del conjunto de los inventarios. Con todo, las diferencias finales del INFIT no son significativas puesto que, en el sumatorio, la distancia sólo puede ser de 10,5 puntos entre Q12 (31 puntos) y Q5 (41,5 puntos).

En cuanto al segundo gran grupo de criterios de raigambre natural, se observan puntuaciones, en general, bastante altas, en comparación con las registradas en ámbitos como el ibérico, la zona boreal escandinava, la Patagonia o las unidades estudiadas en los Balcanes. De hecho, muchas de estas parcelas registran las puntuaciones más altas, fundamentalmente centradas en las especies endémicas. El grado de endemismos de la región mediterránea chilena es uno de los más altos a escala planetaria a excepción de las islas pero, además, el bosque esclerófilo mediterráneo con palmeras cuenta con puntuaciones muy elevadas como las registradas en Q1, Q2, Q4 y Q5 (todas con 7 puntos, siendo el máximo 10). Especies como la propia palma chilena (Jubaea chilensis), pero también: Peumus boldus, Quillaja saponaria, Cryptocarya alba, Aristiguieta salvia, Tropaelum ciliatum, Calceolaria segethii, Alstroemeria pulchra, Conyza hirtella, Stachys grandidentata, Calceolaria morisii, Blechnum hastatum, Chusquea cumingii, Retanilla trinervia... son o bien endemismos amplios del territorio chileno o endemismos de la región central de Chile que, en gran medida, coincide con la zona de ámbito mediterráneo.

Otro de los criterios con puntuaciones bastante elevadas sería el de rareza. Se trata de plantas catalogadas como tal por distintas fuentes. La propia Jubaea chilensis es calificada como escasa $\mathrm{y}$, además, genera una formación que es considerada como rara y muy restringida, lo que le dota de un valor muy considerable. Otras especies consideradas como raras y vulnerables serían: Cuscuta chilensis, Calceolaria segethii, Conyza hirtella, Adesmia arborea, etc. Este criterio es el más importante y, por eso, aparece multiplicado por 2. En cualquier caso, las puntuaciones registradas se mueven entre los 14 puntos de Q6 y Q12 (donde menor cobertura alcanzan las palmas, curiosamente) y los 5 de Q8, en realidad unas diferencias bastante notables. Junto a estos criterios, otros como el grado de relictismo y el carácter finícola, tanto de las diferentes formaciones o facies como de las plantas que las integran, muestran puntuaciones modestas y perfectamente equiparables a otras registradas en otros ámbitos como los citados anteriormente. El palmeral en sí es considerado como relictual de manera que la mayor parte de los inventarios reciben puntos por esta cuestión dependiendo de la cobertura o abundancia de esta especie. En lo que respecta al último de los criterios, el que valora el carácter finícola, 
lo cierto es que existen muy pocas plantas que puedan considerarse como tal y el propio palmeral, en esta zona, se encontraría en el centro de su área, con lo que las puntuaciones son bajas para todas las parcelas. Con todo, las diferencias dentro del interés territorial global son bastante acusadas entre Q6 con 24,5 puntos o Q12 con 23,5 y Q8 que registra las cifras más bajas con tan sólo 8,5 puntos. Mientras los dos primeros son palmerales muy abiertos puesto que los pies de Jubaea chilensis son escasos y muestran bajas coberturas, de manera que dan paso a otras especies de arbustos pero fundamentalmente herbáceas, el último responde a un palmeral con una cierta cobertura de eucaliptos que empobrece el complejo.

En cuanto a los criterios de naturaleza mesológica, lo cierto es que lo elevado de las pendientes, la escasez de la capa edáfica y la función contra los procesos de erosión que ejerce este tipo de formación, generan importantes puntuaciones tanto para el criterio geomorfológico (el más importante $\mathrm{y}$, por tanto, multiplicado por 2) como para el edáfico. En estos dos criterios la valoración fundamental viene de manos de la cantidad de suelo desnudo que se puede encontrar y de la potencialidad para reciclar o mineralizar la materia orgánica. En todas las parcelas, incluso teniendo en cuenta que los incendios son muy frecuentes, las coberturas son elevadas y, con ello, las puntuaciones para estos dos criterios. En el primer caso oscila entre los 16 puntos dados a aquellas parcelas con vegetación arbórea rala pero sotobosque denso y los 18 dados a la vegetación arbórea densa con coberturas de sotobosque medias. En lo que respecta al criterio edafológico, las parcelas oscilan entre los 7 puntos otorgados a las formaciones arbustivas o arborescentes con tasa media de producción, retención o reciclabilidad de materia orgánica y los 8 puntos que suponen la misma formación pero, en este caso, con facies arbóreas claras y netas.

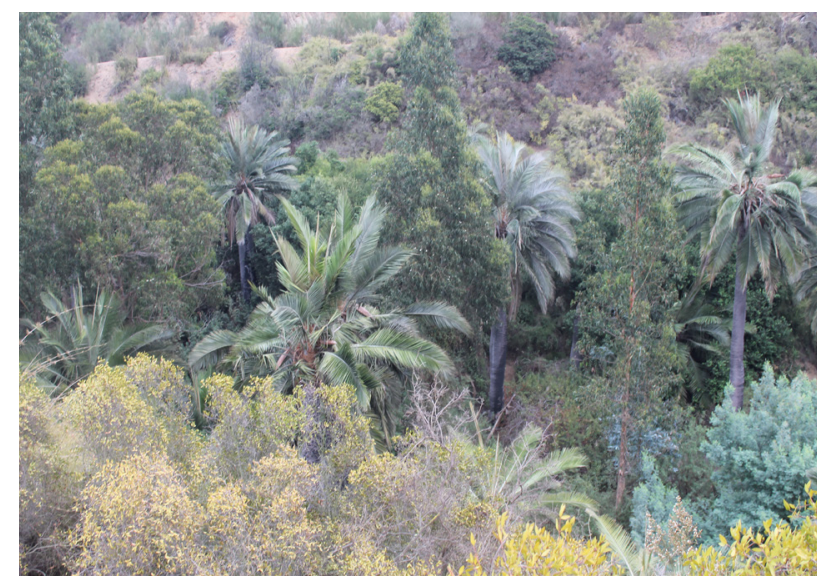

Figura 6: Foto de especies alóctonas que componen el cortejo florístico de la palma chilena y entre las cuales destaca la mimosa Acacia dealbata, entre otras especies.

Figure 6: Picture of allocthonous species that compose the floristic entourage of the Chilean palm, with the Acacia dealbata mimosa, among other species, standing out.
Otros criterios mesológicos son el climático que mide la capacidad de generar condiciones microclimáticas de temperatura o humedad. En este caso las puntuaciones son muy iguales oscilando entre los 7 puntos asignados a la vegetación arbórea rala o plantaciones intensivas (Q7 y Q8), los 8 puntos a las facies arbóreas ralas con sotobosque denso y los 9 a la facies contraria; vegetación arbórea densa con sotobosque un poco más ralo (Q3 y Q4). La función hidrológica, por su parte, valora la capacidad de las formaciones analizadas para asegurar una correcta circulación hídrica y una capacidad de almacenamiento del agua en el suelo o la roca. En este caso las puntuaciones tampoco fluctúan excesivamente de manera que oscilan entre los 8 puntos otorgados a Q6, Q7 y Q8 y el resto con 9 puntos. Por último, la función faunística mide la capacidad de dar cobijo, albergar, ofrecer recursos, etc. a los grupos faunísticos. En este caso se mide la capacidad de generar recursos tróficos, la estructura más o menos intrincada y diversa, etc. Tampoco para este criterio las puntuaciones oscilan en exceso. Lógicamente, aquellas parcelas con plantaciones o abundancia de especies alóctonas muestran puntuaciones más bajas (Q7, Q8, Q9 y $\mathrm{Q} 10)$, mientras que Q11, curiosamente y aunque muestra una cierta cobertura de mimosa, muestra la mayor de las puntuaciones (8 puntos) al contar con una estructura diversa, en parte con forma de dehesa y con especies muy dadas a generar frutos atractivos para la fauna, especialmente las aves.

Las diferencias en el sumatorio de los distintos subcriterios y que dan lugar al interés mesológico global (INMES) oscilan muy poco entre los 51 puntos de Q3, Q4 y Q11 y los 44 de Q7 y Q8. Lógicamente, estas dos parcelas responden a una plantación forestal con eucaliptos más intensiva generando puntuaciones mucho más bajas para este grupo de criterios. En el caso de Q8 sus puntuaciones altas generales $\mathrm{y}$, especialmente la registrada para el subfactor faunístico, la hacen acreedora de grandes puntuaciones.

Con todo, el INNAT global registra la suma de los distintos criterios naturales y nos otorga una jerarquía clara entre las puntuaciones más altas: Q5 con 112,5 y Q1-Q2 con 112 puntos y, por la parte baja, Q8 con 81,5 y Q7 con 86. Como se observa, las diferencias comienzan a ser considerables puesto que entre la puntuación más alta y la más baja se dan diferencias de 31 puntos.

En cuanto a los criterios relacionados con la parte cultural; etnográfica, manejo, gestión, percepción, capacidad pedagógica, etc. Lo cierto es que las diferencias son relativamente modestas debido a que el área es bastante restringida y no existen grandes contrastes de cariz cultural o de gestión. En cualquier caso, es reseñable que las valoraciones culturales vienen a refrendar las líneas básicas apuntadas para los criterios naturales. En general muestran valores más elevados las parcelas o inventarios con mayor grado de naturalidad que aquellas que responden a las facies de repoblación con especies alóctonas. De esta manera, los valores del INCUL global oscilan entre los 9 puntos de Q8, el más bajo y los 16 de Q3, Q4, Q9, Q11 y Q12. 
La suma de los criterios naturales y culturales da lugar al siguiente ranquin (INCONTFOR): en la parte superior y con puntuaciones altas se situarían, por este orden: Q5 (156 puntos), Q12 (153,5 puntos), Q2 (151 puntos) y Q9 (151 puntos). Por la parte baja, y a gran distancia, se situarían Q7 y Q8 ambos con 124 puntos. El resto de parcelas se situarían mucho más cerca de la parte alta que de la parte baja, es decir, los palmerales que se ven expuestos a una cierta cobertura con eucaliptos muestran unas puntuaciones notablemente más bajas que el resto. Curiosamente, la facies con sólo mimosa como especie alóctona se encuentra con puntuaciones medias a altas.

Los valores del INCONTFOR (criterios naturales y culturales) son muy similares a los obtenidos en otros ámbitos con formaciones que posteriormente obtuvieron puntuaciones altas. Estarían a la altura de algunas formaciones ibéricas y de los Balcanes, sin embargo, superan a éstas en los valores naturales aunque son más pobres en los valores culturales.

Por último, a estas puntuaciones habría que multiplicar el factor global de amenaza que se registra a partir de tres subcriterios como son la densidad de población, la accesibilidad-transitabilidad y el coeficiente de amenaza alternativa. Va a ser en este grupo de criterios donde, realmente, el palmeral de Jubaea chilensis, va a concentrar las máximas puntuaciones obtenidas hasta la fecha. Lo cierto es que estos tres criterios muestran puntuaciones muy elevadas en general. La explicación es sencilla: el primer criterio, el de densidad poblacional, es el mismo para todos puesto que, en este entorno, se registra una densidad global de más de $450 \mathrm{hab} . / \mathrm{km}^{2}$. Esta gran presión poblacional ya supone una gran amenaza para la quebrada El Quiteño. Por si eso fuera poco, aunque el carácter intrincado de las distintas facies hace que esta unidad, en general, sea poco transitable, la existencia de una autopista de gran capacidad y una serie de carreteras de distinta capacidad, garantiza una accesibilidad muy alta. La combinación de estos dos parámetros dentro de la matriz de doble entrada nos da índices que oscilan entre los 4 y los 6 puntos. Es decir, índices de accesibilidad-transitabilidad medios a altos. Por último, el coeficiente de amenaza alternativa también es muy alto en general. La existencia de incendios devastadores y recurrentes (prácticamente todos los años), la constatación de vertidos de diversa naturaleza, la introducción de un gran número de neófitos, la existencia de algunas construcciones y tendidos eléctricos amén de otras dotaciones e infraestructuras, generan unas puntuaciones muy altas per se. Por si esto fuera poco, la no existencia de una figura de protección y el descontrol institucional da lugar a la colonización de amplios sectores de los alrededores de Valparaíso para la urbanización ilegal. Todo ello genera puntuaciones de riesgo muy altas o absolutas ( 9 y 10 puntos).

Con todo, el factor global de amenaza se acerca a su máximo situado en 30 puntos. En las parcelas estudiadas oscila entre los 24 puntos de Q3, Q6, Q10 y Q11 y los 26 puntos de Q2 y Q7. El resto de las parcelas contarían con 25 puntos. En ninguno de los ámbitos estudiados hasta la fecha se había llegado a registros tan altos. Es precisamente esta cuestión la que otorga esas cifras tan elevadas a esta unidad, de manera que marca los récords absolutos de puntuación a escala global.

De esta forma, la prioridad de conservación (PRI$\mathrm{CON}$ ) genera la siguiente prelación: las parcelas más valoradas son Q2 (3926 puntos), Q5 (3900 puntos), Q12 (3837,5 puntos) y Q9 (3775 puntos). Todas ellas responden a un palmeral de sotobosque esclerófilo a excepción de Q9 que, curiosamente, se corresponde con un palmeral pero con eucalipto y mimosa. Puede considerarse que la existencia de un dosel arbóreo con gran cobertura y una gran diversidad de especies garantiza, precisamente, estas elevadas cifras. En la parte baja se situarían Q8 (3100 puntos), Q7 (3224 puntos), ambos palmerales pero con repoblaciones intensivas de eucalipto y Q3 (3492 puntos); respondiendo a una facies pobre de palmeral con sotobosque esclerófilo ralo. De hecho, la cobertura y número de palmeras en esta parcela es baja. Por último, (Q10), una facies con acacia y eucalipto mostrando además buenas coberturas, se situaría en cuarta posición por la cola con 3504 puntos.

En relación o comparación con unidades de vegetación del entorno mediterráneo de Chile, se debería resenar que obtienen puntuaciones muy similares a excepción del factor global de amenaza que dispara el bosque esclerófilo mediterráneo con palmeras. También con altas cifras aparecerían, por este orden, el bosque de roble de Pellín (1849,2 puntos), el bosque laurifolio esclerófilo (1445,5 puntos) y, muy cercanos en puntuación: el bosque de ciprés de la Cordillera (1383,3 puntos) y el bosque laurifolio hidrófilo (1367,5 puntos). En cualquier caso, hay que destacar que la prioridad de conservación en estos sectores se encuentra muy influenciada por la existencia de una amenaza alternativa muy clara, como es el fuego (Castillo \& Quintanilla, 2007). En el lado opuesto se encontraría el matorral mediterráneo esclerófilo, con unas puntuaciones muy modestas que justo rebasan los 500 puntos. En este caso, el riesgo de incendios es, si cabe, más elevado; pero las escasas puntuaciones registradas en el resto de parámetros y subcriterios hace que, en general, muestre cifras ciertamente pequeñas. También el bosque de roble de Santiago muestra valores bastante discretos (1275 puntos); pero en este caso, se debe a sus menores posibilidades de incendio, determinadas por un carácter no pirófito de la comunidad, en general y, sobre todo, por ocupar altitudes y sectores mucho más protegidos de la presencia sistemática de los incendios forestales.

Para concluir, hay que reseñar que en el ámbito concernido en el presente trabajo aparecen valoraciones muy elevadas en relación con espacios y unidades estudiados por nuestro equipo de investigación no sólo a nivel europeo sino también en el propio territorio chileno. Así, entre el bosque mediterráneo de palmas y el matorral mediterráneo esclerófilo hay una distancia de nada menos que 3000 puntos; en tanto que en la Patagonia Chilena (con datos y resultados valorativos que, de momento, permanecen inéditos) la diferencia entre las unidades más valoradas, caso del bosque mixto Cohigual-Lengar (Nothofa- 
gus betuloides-Nothofagus pumilio) con 2159 puntos, y la menos valorada, el matorral de mata negra (Mulinum spinosum) con 1238,4 puntos, existe una diferencia de sólo 922,6 puntos. También dentro de Patagonia nuestro equipo de investigación ha podido valorar unidades como el matorral de notro (Embotrium coccineum), con 1725,5 puntos o el bosque de ñirre (Nothofagus antarctica) con 1659,8 .

Por cotejar estos datos del Chile mediterráneo con los obtenidos por nuestro equipo de trabajo en territorio europeo, ninguna unidad analizada llega a sobrepasar en este último ámbito geográfico los 3000 puntos. Y, desde luego, no se llegan a registrar tan fuertes diferencias entre unidades. Por poner varios ejemplos, en la región mediterránea de la península Ibérica el robledal de Quercus pyrenaica puede alcanzar los 1430 puntos, el quejigal de Quercus faginea los 1380 y el encinar de Quercus rotundifolia los 1365. En otros sectores ibéricos, como el Atlántico, el alcornocal de Quercus suber llega a situarse en los 2623,9 (el valor más elevado que había sido registrado hasta el momento por nuestro equipo), el tremolinar de Populus tremula en los 2384,5, el robledal de Quercus robur los 2383,5 y el argomal-matorral de Ulex europaeus 1555 puntos.

\section{Conclusiones}

Efectivamente, a la vista del inventariado y la valoración sistemática realizada, el bosque esclerófilo mediterráneo con palma chilena registra puntuaciones medias de 3594,6 puntos, aunque hay parcelas que pueden llegar a los 3926 puntos.

En general, en todos los parámetros, las facies con mayor grado de naturalidad muestran puntuaciones más elevadas que aquellas que albergan especies exóticas, fundamentalmente Eucalyptus globulus.

Las puntuaciones registradas en el INNAT son bastante similares a otras formaciones chilenas y a escala global, si bien, esta formación de bosque esclerófilo mediterráneo con palma chilena muestra unas puntuaciones muy elevadas dentro del criterio del interés territorial global. Más concretamente, muestra cifras muy elevadas dentro de los subcriterios de endemicidad y de rareza.

En lo que respecta al criterio mesológico, estas formaciones cuentan con buenas puntuaciones en criterios como el geomorfológico y el edáfico, de hecho, muestran una gran capacidad de retención de la materia orgánica y el suelo, de manera que evitan la pérdida de la capa edáfica y los procesos de erosión, muy importantes en la zona que cuenta con un régimen precipitacional de carácter torrencial.

En cuanto a los criterios culturales, al contrario que ocurría con los naturales, muestran unas puntuaciones muy discretas. La percepción y el potencial pedagógico son medios a bajos, mientras que elementos culturales como ciertos aterrazamientos y algunas construcciones rústicas son las únicas cuestiones que deberían ser tenidas en cuenta para la gestión de este espacio.
Donde verdaderamente se disparan las puntuaciones son en el factor global de amenaza que fluctúa entre 24 y 26 puntos siendo el máximo potencial de 30 puntos. La gran densidad de población junto a los incendios, la introducción de xenófitas, la construcción y urbanización descontrolada y la aparición de determinadas dotaciones e infraestructuras, da lugar a un PRICON tan elevado como el obtenido.

De cara a la planificación y gestión de este espacio y estos paisajes forestales es absolutamente indispensable poner freno a las amenazas antes apuntadas a partir de planificaciones de conservación ad hoc y la puesta en marcha de un Plan Rector de Uso y Gestión similar a aquellos que se desarrollan para los parques naturales o los biotopos protegidos en el ámbito europeo. La administración chilena cuenta con suficientes tipologías de espacios protegidos y herramientas político-administrativas para, con urgencia, abordar la conservación, recuperación y restauración del paisaje de palmeral con bosque esclerófilo mediterráneo. Junto a ello es absolutamente estratégica la existencia de una labor policial que controle las actividades no permitidas.

A la vista de la diferencia entre las facies con y sin plantaciones de exóticas, sería recomendable la redacción de una planificación para la erradicación de las xenófitas que se llevara a cabo de forma sistemática y lo más urgentemente posible.

Finalmente, es interesante volver a destacar el elevado valor global final (PRICON). Es muy superior a paisajes del ámbito mediterráneo chileno, de la Patagonia o de los ámbitos europeos estudiados y valorados.

\section{Agradecimientos}

A la Universidad de Santiago de Chile (USACH), por la concesión del Proyecto de Investigación con código: 09-1512qp_intexcelenc, Vicerrectoría de Investigación, Desarrollo e Innovación. A los ayudantes de investigación Bryan Lobos y Carolina Santelices.

\section{Referencias}

Álvarez, L., 1964. Geología del área Valparaíso-Viña del Mar. Instituto de Investigaciones Geológicas. Boletín 16, Chile.

Benton, M.J., 2001. Biodiversity on land and in the sea. Geological Journal, 36: 2-4. http://dx.doi.org/10.1002/gj.877.

Cadiñanos, J.A. \& Meaza, G., 1998a. Bases para una Biogeografía aplicada. Criterios y sistemas de valoración de la vegetación. Geoforma Ediciones, 144 pp., Logroño.

Cadiñanos, J.A. \& Meaza, G., 1998b. Nueva propuesta metodológica de valoración del interés y de la prioridad de conservación de la vegetación. Actas del Colloque International de Botanique Pyreneo-Cantabrique, Mauleon.

Cadiñanos, J.A. \& Meaza, G., 2000. Metodología complementaria de evaluación de ecosistemas forestales. Inédito.

Cadiñanos, J.A., Meaza, G \& Lozano, P.J., 2002. Valoración del interés y de la prioridad de conservación de bosques y comunidades preforestales de Larra (Alto Pirineo Navarro). En: La Biogeografia: ciencia geográfica y ciencia 
biológica. Actas del II Congreso Español de Biogeografía. La Gomera.

Cadiñanos, J.A., Díaz, E., Ibísate, A., Lozano, P.J., Meaza, G., Peralta, J., Ollero, A. \& Hormaetxea, O., 2002. Aplicación de una metodología de valoración de la vegetación a riberas fluviales: ensayo en el río Butrón (Bizkaia). Aportaciones geográficas en memoria del Prof. L. Miguel Yetano Ruiz: 65-88 pp., Zaragoza.

Cadiñanos, J.A., Lozano, P.J. \& Quintanilla, V., 2011. Propuesta de marco integrado para la valoración biogeográfica de los espacios Red Natura 2000 de la Comunidad Autónoma del País Vasco. El ejemplo de Gárate-Santa Bárbara. Boletín de la Asociación de Geógrafos Españoles, 57: 33-56.

Costanza, R., d'Arge, R, de Groot, R, Farber, S, Grasso, M., Hannon, B., Limburg, K., Naeem, S., O’Neill, R.V., Paruelo, J., Robert G., Raskin, R.G., Sutton, P. \& van den Belt, M., 1997. The value of the world's ecosystem services and natural capital. Nature, 387: 253-260.

Debinski, D.M., Ray, C. \& Saveraid, E.H., 2001. Species diversity and the scale of the landscape mosaic: do scales of movement and patch size affect diversity? Biological Conservation, 98: 179-190. http://dx.doi.org/10.1016/S00063207(00)00153-1.

Fernández, I., Morales, M., Olivares, L., Salvatierra, J., Gómez, M. \& Montenegro, G., 2009. Restauración ecológica para ecosistemas nativos afectados por incendios forestales. PUCCH. Facultad de Agronomía. 161pp., Santiago.

Figueroa, H., \& Portal, E., 1997. Geomorfología de los riesgos naturales en la cuenca del estero Marga Marga, Viña del Mar. Revista Geográfica de Valparaiso, 28: 77-107.

Flores, L. \& Aguirre, F., 2008. Riqueza florística del Santuario de la Naturaleza Palmar El Salto, Viña del Mar, Región de Valparaíso, Chile. Gayana, Botánica, 65 (1): 33-45.

Grau, J., 2004. Palmeras de Chile. Ediciones OIKOS 203 pp., Santiago.

Lozano, P.J., Cadiñanos, J.A., Longares, L.A. \& Cid, M.A., 2007. Valoración Biogeográfica de los tipos de bosque en la combe de Huidobro (Parque Natural de las Hoces del EbroBurgos). Actas del $4^{\circ}$ Congreso Español de Biogeografia, 19, Ávila.

Lozano, P.J. \& Cadiñanos, J.A., 2009. Propuesta de marco metodológico integrado para la valoración de Espacios de la Red Natura 2000 de la Comunidad Autónoma del País Vasco. El ejemplo de Gárate-Santa Bárbara (País Vasco). Biogeografía Scientia Biodiversitatis: 199-206 pp., Málaga.

Lozano, P.J., Cadiñanos, J.A., Latasa, I. \& Meaza, G., 2013. Caracterización y valoración biogeográfica de los pinares de Pinus uncinata del karst de Larra (Alto Pirineo Navarro) para su ordenación y gestión, Geographicalia, 63-64: 95-120.

Lozano, P.J., Cadiñanos, J.A., Latasa, I., Quintanilla, V., \& Meaza, G., 2015. Caracterización, valoración y evaluación de los paisajes vegetales de Chile Mediterráneo. Boletín de la Asociación de Geógrafos Españoles, 67: 14-32.

Luzio, W., 2010. Suelos de Chile. Universidad de Chile. 301 pp. Santiago.

Mc.Neill, J.R., 2000. Algo nuevo bajo el sol. Historia medioambiental del mundo en el siglo XX. Alianza ensayo, $504 \mathrm{pp}$, Madrid.

Meaza, G., Cadiñanos, J.A. \& Lozano, P.J., 2006. Valoración biogeográfica de los bosques de la reserva de la biosfera de Urdaibai (Vizcaya). Actas del III Congreso Español de Biogeografia: 399-411 pp., Urdaibai.

Möder, L., Rojas, O. \& González, L., 1997. Reestudio de los límites propuestos para creación del área "Palmar El Salto" como Santuario de la Naturaleza. Informe técnico $\mathrm{N}^{\mathrm{o}} 15 / 97$. U.G. Patrimonio Silvestre CONAF V Región. $6 \mathrm{pp}$.

Novoa, R. \& Villaseca, S., 1989. Mapa Agroclimático de Chile (Escala 1:1.000.000). Instituto de Investigaciones Agropecuarias. Facultad de Agronomía. Universidad de Chile, 212 pp. Santiago.

Quintanilla, V. \& Castro, R., 1998. Seguimiento de las cubiertas vegetales postincendios forestales, en la zona mediterránea costera de Chile. Serie Geográfica: 147-154.

Quintanilla, V. \& Reyes, C. 1999. Modificaciones por efecto del fuego en el bosque esclerófilo de quebradas húmedas de Chile central y su incidencia en la palma chilena. Revista Geográfica Terra Australis, 44: 7-18.

Quintanilla, V. \& Castillo, M. 2009. Degradación de la palma más austral del mundo acelerada por los fuegos estivales en cordones litorales de Valparaíso y Viña del Mar. Un caso de perturbación permanente. Revista Investigaciones Geográficas, 41: 41-54.

Quintanilla, V., Cadiñanos, J.A., Latasa, I. \& Lozano, P.J. 2012. Aproximación biogeográfica a los bosques de la región mediterránea de Chile: caracterización e inventario. Boletín de la Asociación de Geógrafos Españoles, 60: 91-114.

Quintanilla, V. \& Morales, M., 2013. Perturbaciones de los fuegos de verano en la palma más austral del mundo (Jubaea chilensis (Moll)) Baillon en microcuencas de la zona mediterránea de Chile. Cuadernos Geográficos, 52: 129-152.

Sagastibeltza, E., Lozano P.J., Herrero, X., 2014. Nafarroako Bortzirietako baso-landaredien paisaien inbentariazioa, karakterizazioa eta balorazio biogeografikoa. Lurralde, 37 : 97-133.

Strijker, D., Sijtsma, F.J. \& Wiersma, D., 2000. Evaluation of nature conservation: An application to the Dutch Ecological Network. Environmental and Resource Economics, 16: 363-378.

Whittaker, R.H., 1972. Evolution and Measurement of Species Diversity. International Association for Plant Taxonomy, 21: 213-251. http://dx.doi.org/10.2307/1218190. 